\title{
Controlling the cation distribution and electric polarization with epitaxial strain in Aurivillius-phase $\mathrm{Bi}_{5} \mathrm{FeTi}_{3} \mathrm{O}_{15}$
}

\author{
Axiel Yaël Birenbaum* and Claude Ederen \\ Materials Theory, ETH Zürich, Wolfgang-Pauli-Strasse 27, 8093 Zürich, Switzerland
}

(Dated: November 6, 2018)

\begin{abstract}
This work explores the impact of in-plane bi-axial (epitaxial) strain on the cation distribution and electric polarization of the Aurivillius-phase compound $\mathrm{Bi}_{5} \mathrm{FeTi}_{3} \mathrm{O}_{15}$ using first-principles electronic structure calculations. Our calculations indicate that the site preference of the $\mathrm{Fe}^{3+}$ cation can be controlled via epitaxial strain. Tensile strain enhances the preference for the inner sites within the perovskite-like layers of the Aurivillius-phase structure, whereas compressive strain favors occupation of the outer sites, i.e., the sites close to the $\mathrm{Bi}_{2} \mathrm{O}_{2}$ layer. Controlling the distribution of the magnetic cations offers the possibility to control magnetic order in this magnetically dilute system. Furthermore, the magnitude of the electric polarization is strongly strain-dependent, increasing under tensile strain and decreasing under compressive strain. We find strongly anomalous Born effective charges, both of the $\mathrm{Bi}^{3+}$ and the $\mathrm{Ti}^{4+}$ cations.
\end{abstract}

Controlling the properties of complex transition metal oxides by epitaxial strain, i.e., by growing thin films of a certain material on a substrate with specific lattice mismatch, has emerged as a very efficient way for designing optimized functionalities ${ }^{11}$ In particular, the effect of epitaxial strain on the ferroelectric properties of perovskite materials is well studied ${ }^{2}$ and dramatic enhancements of polarization and ferroelectric ordering temperatures,, 3 as well as emergence of ferroelectricity in otherwise nonpolar materials have been reported ${ }^{4 / 5}$

Recently, layered perovskite-related systems have come into focus as being potentially more amenable to developing polar lattice distortions compared to bulk perovskites ${ }^{6}$ Examples include artificial perovskite superlattices and double perovskites, as well as several families of naturally-layered perovskite-derived crystal structures such as the Ruddlesden-Popper series, Aurivillius-phases, or Dion-Jacobson systems. Only few studies addressing the strain response of these naturally-layered materials are currently available. Such studies are, however, of great interest due to the different mechanism underlying the ferroelectricity in these systems, which could lead to a different strain response compared to bulk perovskite ferroelectrics.

Here we study the case of $\mathrm{Bi}_{5} \mathrm{FeTi}_{3} \mathrm{O}_{15}$ (BFTO), a representative of the family of naturally-layered Aurivillius-phases, which is of particular interest due to its potential multiferroic properties $\frac{9}{9}$ The crystal structure of the Aurivillius-phases consists of $m$ perovskite layers $\left(\mathrm{A}_{\mathrm{m}-1} \mathrm{~B}_{\mathrm{m}} \mathrm{O}_{3 \mathrm{~m}+1}\right)^{2-}$ stacked periodically along the [001] direction, and separated by fluorite-like $\left(\mathrm{Bi}_{2} \mathrm{O}_{2}\right)^{2+}$ layers (see Fig. 11) ${ }^{10 \mid 11}$ BFTO corresponds to the case with $m=4$.

A previous first-principles study of polarization-strain coupling in the $m=3$ Aurivillius compound $\mathrm{Bi}_{4} \mathrm{Ti}_{3} \mathrm{O}_{12}$ has shown a large response of in-plane polarization under bi-axial strain! $\frac{13}{}$ Apart from the introduction of an additional perovskite layer and the presence of the nominally non-ferroelectric $\mathrm{Fe}^{3+}$ cation, an important additional degree of freedom in BFTO compared to $\mathrm{Bi}_{4} \mathrm{Ti}_{3} \mathrm{O}_{12}$ is the distribution of $\mathrm{Fe}^{3+}$ and $\mathrm{Ti}^{4+}$ cations over the available $B$
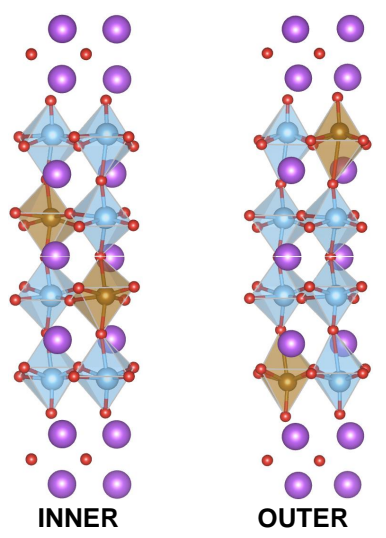

$\mathrm{Bi}\left(\mathrm{Bi}_{2} \mathrm{O}_{2}\right)$
$\mathrm{Bi}\left(\mathrm{Bi}_{2} \mathrm{O}_{2}\right)$
$\mathrm{B}(0)$
$\mathrm{Bi}$ (perov.)
$\mathrm{B}$ (i)
$\mathrm{Bi}$ (perov.)
$\mathrm{B}$ (i)
$\mathrm{Bi}$ (perov.)
$\mathrm{B}(\mathrm{o})$
$\mathrm{Bi}\left(\mathrm{Bi}_{2} \mathrm{O}_{2}\right)$
$\mathrm{Bi}\left(\mathrm{Bi}_{2} \mathrm{O}_{2}\right)$

FIG. 1. (Color online) The two $B$-site cation distributions used in this work, inner and outer. Sites occupied with Fe (Ti) are indicated as brown (blue) spheres with corresponding coordination octahedra; $\mathrm{Bi}(\mathrm{O})$ ions are shown as purple (red) spheres. Labels for the cations in the different layers (used for the discussion of Born effective charges) are listed on the right. This figure was constructed using VESTA ${ }^{12}$

sites within the perovskite layers of the Aurivillius structure. While the $\mathrm{Fe}^{3+} / \mathrm{Ti}^{4+}$ cations do not tend to form an ordered arrangement, a preferential occupation of the inner perovskite site with $\mathrm{Fe}$ has been reported experimentally ${ }^{14}$ and confirmed recently by density functional theory (DFT) calculations. $\frac{9}{9}$ The cation distribution can in principle influence properties such as electric polarization or the likelihood and character of magnetic order, and it could also affect the strain response of the material. Furthermore, a systematic variation of the relaxed lattice constants with the distribution of Fe over the inner/outer perovskite layers has been found in Ref. 9, suggesting that it might be possible to influence the cation distribution by epitaxial strain. Here, we verify this hypothesis and study the effect of strain on the cation distribution and on the resulting electric polarization of BFTO.

We use a unit cell that corresponds to the primitive unit cell of the experimentally-observed $A 2_{1}$ am struc- 
ture of BFTO, containing two formula units. There are 10 symmetry-inequivalent ways to distribute $2 \mathrm{Fe}$ and 6 Ti atoms over the 8 available peroskite $B$ sites within this unit cell ${ }^{[9}$ We focus on two representative configurations depicted in Fig. 1 one with all Fe sitting in the outer perovskite layers and one with all Fe sitting in the inner perovskite layers (denoted as $\mathrm{O} 1$ and I1 in Ref. (9). For both configurations, the specific distribution of $\mathrm{Fe}^{3+}$ and $\mathrm{Ti}^{4+}$ cations lowers the experimentally observed $A 2_{1}$ am space group symmetry to monoclinic $P 2_{1}$. Both configurations allow to define a centrosymmetric reference structure with $P 2_{1} / m$ symmetry for calculating the spontaneous electric polarization by removing all structural distortions that break inversion symmetry.

In order to model the elastic boundary conditions corresponding to the epitaxial constraint imposed by a substrate, we fix the two short in-plane lattice parameters, $a$ and $b$, to be equal to an effective substrate lattice constant $a^{\prime}$, and the corresponding lattice vectors to form a $90^{\circ}$ angle. This corresponds to a thin-film/substrate interface forming a square lattice. Furthermore, for simplicity we constrain the out-of-plane lattice vector to correspond to a perfect base-centered orthorhombic Bravais lattice, in spite of the lower monoclinic symmetry of the whole structure. We find the optimal value of the out-ofplane lattice parameter $c$ (in our notation corresponding to the conventional orthorhombic Bravais lattice) by relaxing all ionic positions for fixed $a^{\prime}$ and different values of $c$. In the following, whenever comparison with experimental structural data is made, Ref. 8 is used, and we define $0 \%$ strain relative to the average experimental inplane lattice constant $a_{0}^{\prime}=\left(a^{\exp }+b^{\exp }\right) / 2=5.45 \AA$.

We perform DFT calculations using the projector augmented wave (PAW) method as implemented in the Vienna $a b$ initio simulation package (VASP) $\stackrel{15 / 16}{ }$ and the generalized gradient approximation according to Perdew, Burke, and Ernzerhof optimized for solids (PBEsol) ${ }^{17}$ Our PAW potentials include 15 valence electrons for $\mathrm{Bi}\left(6 s^{2} 5 d^{10} 6 p^{3}\right), 14$ for $\mathrm{Fe}\left(3 p^{6} 4 s^{2} 3 d^{6}\right), 10$ for $\mathrm{Ti}$ $\left(3 p^{6} 4 s^{2} 3 d^{2}\right)$, and 6 for $\mathrm{O}\left(2 s^{2} 2 p^{4}\right)$. We include a Hubbard " $+U$ " correction with $U_{\text {eff }}=3.0 \mathrm{eV}$ to correctly treat the strong interactions between the Fe $d$ electrons. ${ }^{[18}$ Ionic positions are relaxed until the residual forces are smaller than $10^{-3} \mathrm{eV} / \AA$. Calculations are converged using a $\Gamma$ centered $k$-point mesh with $4 \times 4 \times 2$ divisions along the three reciprocal lattice vectors and a plane wave cutoff energy of $550 \mathrm{eV}$. As shown previously ${ }^{[9}$ all magnetic couplings in BFTO are antiferromagnetic, hence we fix antiparallel orientation of the magnetic moments of the two $\mathrm{Fe}^{3+}$ cations within the unit cell.

Fig. 2 shows the optimized out-of-plane lattice parameter $c$ as well as the energy of both inner and outer configurations for a range of in-plane lattice parameters $a^{\prime}$. The minimum of the energy for each configuration indicates the preferred in-plane lattice parameter for that configuration. Thus, it can be seen that the inner configuration prefers a larger in-plane lattice constant $a^{\prime}$ than the outer configuration and a shorter out-of-plane lat-

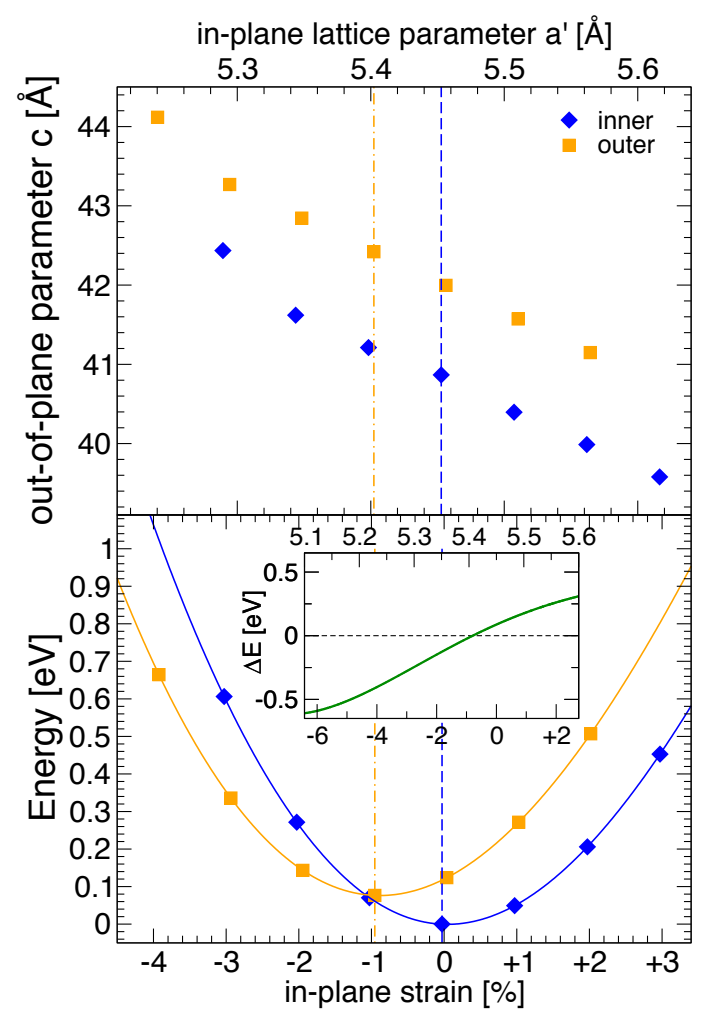

FIG. 2. (Color online) Out-of-plane lattice parameter $c$ (top) and total energy per unit cell (bottom) as a function of inplane biaxial strain (with the axis at the top of the graph giving the corresponding in-plane lattice parameter), for the inner (blue diamonds) and outer (orange squares) configuration. The dashed lines mark the corresponding equilibrium in-plane parameters for the inner (blue) and outer (orange) configurations, respectively. The inset shows the energy difference $\Delta E$ (per unit cell) between the outer and inner configuration for different values of in-plane strain.

tice parameter $c$, in agreement with the bulk relaxations presented in Ref. 9. We note that the position of the energy minimum for the inner configuration, i.e., the corresponding preferred in-plane lattice parameter, agrees very well with the averaged experimental in-plane lattice constant, which serves as our zero strain reference. For a given in-plane lattice parameter, the outer configuration always leads to a larger out-of-plane lattice parameter than the inner configuration. This is due to the strong local tetragonality around the $\mathrm{Fe}^{3+}$ cation occupying an outer site ${ }^{9}$ Furthermore, it can be seen that the energy minimum for the inner configuration is lower than the energy minimum for the outer configuration, consistent with the inner site preference of $\mathrm{Fe}^{3+}$ found in Ref. 9.

The inner site preference is increased if $a^{\prime}$ is increased (see Fig. 2, inset). Thus, tensile strain is expected to strengthen the inner site preference. On the other hand, if compressive strain is applied, i.e., for decreasing $a^{\prime}$, the energy difference between inner and outer configuration decreases, and for values below $a^{\prime}=5.40 \AA$, corresponding to about $-1 \%$ strain, the outer configuration has a 


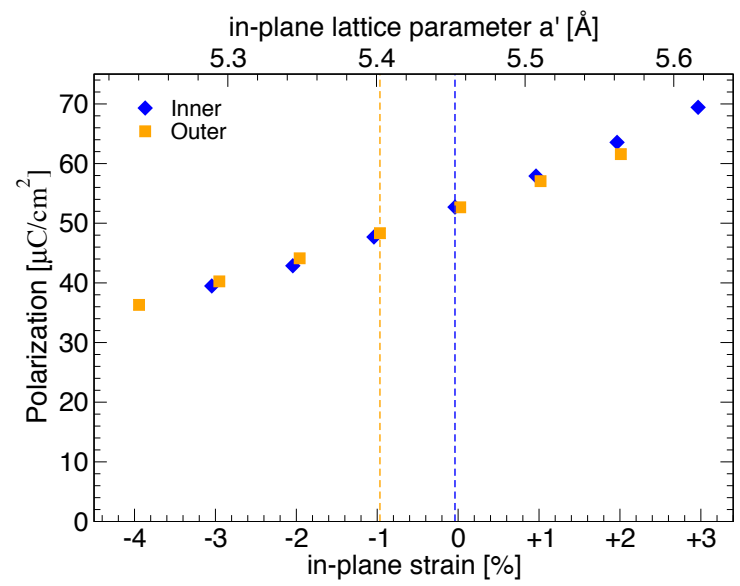

FIG. 3. (Color online) Spontaneous polarization as function of in-plane strain for inner (blue diamonds) and outer (orange squares) configurations. The dashed vertical lines indicate the optimal in-plane lattice parameters for each configuration.

lower energy than the inner configuration.

These results imply that the site preference of the $\mathrm{Fe}^{3+}$ cation can indeed be tuned through epitaxial strain. Tensile strain enforces the preference for the inner sites, while compressive strain can reverse this preference such that the majority of $\mathrm{Fe}^{3+}$ cations occupy outer sites. We note that these results are obtained by considering only two, albeit representative, configurations, and that a more complete treatment would require to consider more configurations. However, the obtained trend is consistent with the observation from Ref. 9 (and Fig. 2) that outer configurations generally favor a more elongated unit cell (along $c$ ) compared to inner ones.

Next, we address the effect of strain on the electric polarization. We calculate the spontaneous polarization $P_{\alpha}$ along a given direction $\alpha$ using Born Effective Charges (BECs), 19

$$
P_{\alpha}=\frac{e}{\Omega} \sum_{\kappa, \beta} Z_{\kappa, \alpha \beta}^{*} u_{\kappa, \beta}
$$

where $\kappa$ indicates the different ions and $u_{\kappa, \beta}$ is the displacement of ion $\kappa$ along direction $\beta$ between the paraelectric reference and the relaxed ferroelectric structure. The BEC tensors are calculated for the polar structures obtained at each strain value using density functional perturbation theory as implemented in VASP.

The calculated polarization as function of strain is depicted in Fig. 3. By symmetry, the polarization is restricted to be aligned along the in-plane two-fold screw axis ( $b$ axis in standard $P 2$ setting, equivalent to the $a$ axis in the experimental $A 2_{1} a m$ structure). At the optimal in-plane lattice constants of each configuration, we find a spontaneous polarization of $P=48.3 \mu \mathrm{C} / \mathrm{cm}^{2}$ for the outer configuration, and $P=52.7 \mu \mathrm{C} / \mathrm{cm}^{2}$ for the inner configuration. These results are consistent with the corresponding bulk values from Ref. 9 ( $P=$
TABLE I. BECs (in units of $|e|$ ) of the different cations for the two cation distributions in the corresponding centrosymmetric $\left(P 2_{1} / m\right)$ and polar $\left(P 2_{1}\right)$ structures. The cations are labeled according to Fig. 1 and separate averages are calculated for the in-plane $(x y)$ and out-of-plane $(z)$ diagonal elements of the BEC tensors.

\begin{tabular}{l|cccc|ccccc}
\hline \hline & \multicolumn{4}{|c|}{ inner } & \multicolumn{4}{c}{ outer } \\
& \multicolumn{2}{|c}{$P 2_{1} / m$} & \multicolumn{2}{c}{$P 2_{1}$} & \multicolumn{2}{c}{$P 2_{1} / m$} & \multicolumn{2}{c}{$P 2_{1}$} \\
& $x y$ & $z$ & $x y$ & $z$ & $x y$ & $z$ & $x y$ & $z$ \\
\hline $\mathrm{Bi}^{3+}\left(\mathrm{Bi}_{2} \mathrm{O}_{2}\right)$ & 4.95 & 4.81 & 4.81 & 4.85 & 5.08 & 4.51 & 4.84 & 4.42 \\
$\mathrm{Bi}^{3+}$ (perov.) & 5.66 & 4.08 & 5.00 & 4.50 & 5.76 & 4.31 & 5.05 & 4.49 \\
$\mathrm{Ti}^{4+}(\mathrm{o})$ & 6.10 & 6.07 & 5.97 & 5.55 & 4.83 & 5.71 & 4.89 & 5.22 \\
$\mathrm{Ti}^{4+}(\mathrm{i})$ & 6.48 & 5.70 & 5.87 & 5.66 & 7.00 & 5.87 & 6.20 & 5.59 \\
$\mathrm{Fe}^{3+}(\mathrm{o})$ & - & - & - & - & 3.49 & 4.57 & 3.55 & 4.05 \\
$\mathrm{Fe}^{3+}(\mathrm{i})$ & 4.75 & 3.90 & 4.52 & 3.77 & - & - & - & - \\
\hline \hline
\end{tabular}

$51.5 / 57.9 \mu \mathrm{C} / \mathrm{cm}^{2}$ for the outer/inner configuration), calculated using the Berry phase method ${ }^{20}$ Note that we are using the BECs calculated for the polar structures to evaluate the spontaneous polarization. Since the BECs decrease when going from the paraelectric to the ferromagnetic structure (see Table I), this leads to a small underestimation of the spontaneous polarization compared to the exact calculation of $P$ using the Berry phase.

We observe that in-plane tensile strain leads to a strong increase in polarization and, conversely, compressive strain leads to a strong decrease. The polarization varies by about $\pm 30 \%$ over the whole strain region considered here, i.e., from $-4 \%$ to $+3 \%$ strain. This trend is similar to many perovskite ferroelectrics, where an elongation (compression) along the polar axis increases (decreases) the ferroelectric displacements of the ions, and thus the polarization, along that direction.21 Similar behavior has also been found in previous DFT studies of the Aurivillius phases $\mathrm{Bi}_{4} \mathrm{Ti}_{3} \mathrm{O}_{12}(m=3)^{13}$ and $\mathrm{SrBi}_{2} \mathrm{Ta}_{2} \mathrm{O}_{9}(m=2) \underline{\underline{24}}$

Furthermore, we can see that for a given in-plane lattice parameter, the calculated polarization is very similar for both configurations, in spite of the differences in the out-of-plane lattice parameter $c$ (see Fig. 2). This indicates that the impact of the $\mathrm{Fe} / \mathrm{Ti}$ distribution on the electric polarization is minimal. The difference in spontaneous polarization obtained for the relaxed inner and outer structures stems mostly from their different in-plane lattice constants $\left(a^{\prime}=5.45 \AA\right.$ and $a^{\prime}=5.40 \AA$, respectively). We can conclude that neither does the presence of the magnetic $\mathrm{Fe}^{3+}$ cations have a negative impact on the overall magnitude of the electric polarization (compared to, e.g., $\mathrm{Bi}_{4} \mathrm{Ti}_{3} \mathrm{O}_{12}$ ), nor does the specific distribution of magnetic cations have a significant effect on this magnitude.

In order to obtain more detailed insights into the ferroelectricity of BFTO, the BECs for the different cations, calculated for the fully relaxed (i.e., without epitaxial constraint) centrosymmetric and ferroelectric states in 
both configurations, averaged over similar (but not necessarily symmetry-equivalent) sites, are presented in Table I. It can be seen that the BECs for both $\mathrm{Bi}^{3+}$ and $\mathrm{Ti}^{4+}$ (and to some extent also for $\mathrm{Fe}^{3+}$ ) are highly anomalous, i.e., they are significantly increased compared to their formal valences. This indicates that displacements of the corresponding cations result in large redistribution of charge and corresponding changes in chemical bonding, $19 \mid 25$ and is usually considered a signature for ferroelectrically-active ions. ${ }^{26 \mid 27}$ The most pronounced difference between the two configurations are the BECs of the $B(\mathrm{o})$ sites in the outer configuration, which are noticeably less anomalous than those of the $B(\mathrm{i})$ sites (and than those of the $B(\mathrm{o})$ sites in the inner configuration). Overall, the BECs for BFTO are quite similar to the BECs calculated for the related $m=3$ Aurivillius phase $\mathrm{Bi}_{4} \mathrm{Ti}_{3} \mathrm{O}_{12}{ }^{13}$ and for the related perovskite system (i.e., $m=\infty) \mathrm{BiFeO}_{3}$. 28

The in-plane BECs of $\mathrm{Bi}^{3+}$ in the perovskite layer are somewhat more anomalous than those in the $\mathrm{Bi}_{2} \mathrm{O}_{2}$ layer and also show a more pronounced reduction when going from the centrosymmetric to the polar structure. This is consistent with the analysis of Withers et al., 29 who suggested the optimization of bond valence sums of the $\mathrm{Bi}^{3+}$ (perov.) cations as driving force for the polar displacements in various Aurivillius systems with $m=1,2$, and 3 . However, a strong reduction of the anomalous in-plane BECs between the centrosymmetric and polar structures can also be observed for the $\mathrm{Ti}^{4+}(\mathrm{i})$ sites, which suggests an active role of the inner $B$ site cations for Aurivillius phases with $m>3$. Furthermore, as first pointed out by Perez-Mato et al., $\frac{30}{30}$ the space group symmetry of the Aurivillius phases allows for coupling between the ferroelectric distortion and two or more octahedral rotation modes, which can give rise to so-called hybrid improper ferroelectricity. ${ }^{31}$ Indeed, a relation between the average tolarance factor of the perovskite blocks, controlling octahedral rotations, and the ferroelectric transition temperature has been empirically found by investigating a large number of Aurivillius

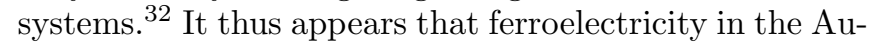
rivillius phases can arise from several factors, which cooperate to give rise to the robust ferroelectric properties observed in this class of compounds.

To summarize, our calculations indicate that it is indeed possible to control the site preference of the $\mathrm{Fe}^{3+}$ cations in BFTO by epitaxial strain. Tensile epitaxial strain is expected to increase the occupation of the inner sites with $\mathrm{Fe}^{3+}$, whereas compressive strain will lead to a preferential $\mathrm{Fe}^{3+}$ occupation of outer sites. In addition, epitaxial strain also provides an efficient way to enhance (or reduce) the magnitude of the spontaneous electric polarization, which, furthermore, is rather insensitive to the actual $B$-site cation distribution.

We point out that the possibility to tailor site preference in BFTO also allows to achieve an essentially homogeneous distribution of $\mathrm{Fe}^{3+}$ cations, i.e., no site preference, under moderate compressive strain. This case could indeed be most favorable for achieving good percolation of magnetic couplings between the $\mathrm{Fe}^{3+}$ ions and thus promoting long range magnetic order. Therefore, controlling the cation distribution through epitaxial strain can provide a way of controlling the multiferroic properties of BFTO and related Aurivillius systems.

\section{ACKNOWLEDGMENTS}

This work was funded by ETH Zurich and the Swiss National Science Foundation under project no. 200021_141357. We thank Nicoló Fanelli and Rolf Homberger for performing some of the initial calculations for this work as part of their undergraduate research project at ETH Zürich.
* yael.birenbaum@mat.ethz.ch

† claude.ederer@mat.ethz.ch

1 D. G. Schlom, L.-Q. Chen, X. Pan, A. Schmehl, and M. A. Zurbuchen, Journal of the American Ceramic Society 91, 2429 (2008)

${ }^{2}$ D. G. Schlom, L.-Q. Chen, C.-B. Eom, K. M. Rabe, S. K. Streiffer, and J.-M. Triscone, Annual Review of Materials Research 37, 589 (2007)

${ }^{3}$ K. J. Choi, M. Biegalski, Y. L. Li, A. Sharan, J. Schubert, R. Uecker, P. Reiche, Y. B. Chen, X. Q. Pan, V. Gopalan, L. Q. Chen, D. G. Schlom, and C. B. Eom, Science 306, 1005 (2004).

4 J. H. Haeni, P. Irvin, W. Chang, R. Uecker, P. Reiche, Y. L. Li, S. Choudhury, W. Tian, M. E. Hawley, B. Craigo, A. K. Tagantsev, X. Q. Pan, S. K. Streiffer, L. Q. Chen, S. W. Kirchoefer, J. Levy, and D. G. Schlom, Nature (London) 430, 758 (2004).

${ }^{5}$ S. Bhattacharjee, E. Bousquet, and P. Ghosez, Phys. Rev.
Lett. 102, 117602 (2009).

6 N. A. Benedek, J. M. Rondinelli, H. Djani, P. Ghosez, and P. Lightfoot, Dalton Trans. 44, 10543 (2015).

7 F. Kubel and H. Schmid, Ferroelectrics 129, 101 (1992).

8 C. H. Hervoches, A. Snedden, R. Riggs, S. H. Kilcoyne, P. Manuel, and P. Lightfoot, Journal of Solid State Chemistry 164, 280 (2002).

9 A. Y. Birenbaum and C. Ederer, Physical Review B 90, 214109 (2014).

10 R. E. Newnham, R. W. Wolfe, and J. F. Dorrian, Mat. Res. Bull. 6, 1029 (1971).

11 B. Frit and J. P. Mercurio, Journal of Alloys and Compounds 188, 27 (1992).

12 K. Momma and F. Izumi, Journal of Applied Crystallography 44, 1272 (2011).

13 S. H. Shah and P. D. Bristowe, Journal of PhysicsCondensed Matter 22, 385902 (2010).

14 N. A. Lomanova, V. G. Semenov, V. V. Panchuk, and 
V. V. Gusarov, Journal of Alloys and Compounds 528, 103 (2012).

15 G. Kresse and J. Furthmüller, Comput. Mat. Sci. 6, 15 (1996).

16 G. Kresse and D. Joubert, Physical Review B 59, 1758 (1999).

17 J. P. Perdew, A. Ruzsinszky, G. Csonka, O. Vydrov, G. Scuseria, L. Constantin, X. Zhou, and K. Burke, Physical Review Letters 100, 136406 (2008).

18 S. L. Dudarev, G. A. Botton, S. Y. Savrasov, C. J. Humphreys, and A. P. Sutton, Phys. Rev. B 57, 1505 (1998).

19 P. Ghosez, J. P. Michenaud, and X. Gonze, Physical Review B 58, 6224 (1998).

20 R. D. King-Smith and D. Vanderbilt, Physical Review B 47, 1651 (1993).

21 C. Bungaro and K. M. Rabe, Phys. Rev. B 69, 184101 (2004).

22 O. Diéguez, S. Tinte, A. Antons, C. Bungaro, J. B. Neaton, K. M. Rabe, and D. Vanderbilt, Phys. Rev. B 69, 212101 (2004).

23 C. Ederer and N. A. Spaldin, Phys. Rev. Lett. 95, 257601 (2005).
${ }^{24}$ Q. Yang, J. X. Cao, Y. Ma, and Y. C. Zhou, AIP Advances 3, 052134 (2013).

25 C. Ederer, T. Harris, and R. Kováčik, Phys. Rev. B 83, 054110 (2011)

${ }^{20}$ M. Posternak, R. Resta, and A. Baldereschi, Physical Review B 50, 8911 (1994).

27 K. M. Rabe and P. Ghosez, in Physics of Ferroelectrics - A modern perspective, edited by K. Rabe, C. H. Ahn, and J.-M. Triscone (Springer, Berlin/Heidelberg, 2007) pp. $117-174$.

28 J. B. Neaton, C. Ederer, U. Waghmare, N. A. Spaldin, and K. M. Rabe, Physical Review B 71, 014113 (2005).

29 R. L. Withers, J. G. Thompson, and A. D. Rae, Journal of Solid State Chemistry 94, 404 (1991).

30 J. Perez-Mato, M. Aroyo, A. Garca, P. Blaha, K. Schwarz, J. Schweifer, and K. Parlinski, Phys. Rev. B 70, 214111 (2004).

31 N. A. Benedek and C. J. Fennie, Phys. Rev. Lett. 106, 107204 (2011).

32 D. Y. Suárez, I. M. Reaney, and W. E. Lee, J. Mater. Res. 16, 3139 (2001). 